\title{
CERTIFICAÇÃO FLORESTAL NO BRASIL - UMA FERRAMENTA EFICAZ PARA A CONSERVAÇÃO DAS FLORESTAS NATURAIS?
}

\author{
Peter Spathelf ${ }^{1}$ \\ Patricia Povoa de Mattos ${ }^{2}$ \\ Paulo César Botosso ${ }^{3}$
}

\begin{abstract}
RESUMO
A certificação florestal é descrita como instrumento de mercado para apoiar um manejo florestal sustentável. Um foco especial é dado pela certificação sob FSC, mas também pelo sistema esquema nacional de certificação florestal no Brasil, o CERFLOR. O que se questiona é se a certificação poderá contribuir com a adoção de práticas de bom manejo pela motivação devido às vantagens advindas da certificação ou pela conscientização do consumidor. As principais forças motrizes do desmatamento nas florestas brasileiras ainda existem, por isso a contribuição da certificação para a conservação das florestas naturais parece ser limitada.

Palavras-chave: certificação florestal, CERFLOR, desmatamento, Brasil
\end{abstract}

\section{FOREST CERTIFICATION IN BRAZIL - AN EFFICIENT TOOL FOR THE CONSERVATION OF NATURAL FORESTS?}

\begin{abstract}
Forest certification is described as a market-oriented tool to support sustainable forest management. Special emphasis is given to forest certification under FSC, but also to the national certification scheme in Brazil, CERFLOR. The key question is, whether forest certification gives a significant contribution to reduce deforestation and conventional unsustainable logging, respectively under the circumstances of the Brazilian forest sector and domestic consumer behaviour. The main driving forces for deforestation in Brazil still exist and, consequently, the impact of forest certification for the conservation of natural tropical forests in Brazil is still seen to be limited.
\end{abstract}

Keywords: forest certification, CERFLOR, deforestation, Brazil

\section{INTRODUÇÃO}

A crescente destruição das florestas tropicais foi o ponto de partida do processo de certificação de florestas nos anos 80 e 90. Consumidores alertados sobre o uso predatório de florestas tropicais aumentaram a pressão sobre as empresas do setor madeireiro para que estas assumissem uma política mais conservacionista e humanista. Medidas de boicote de madeira tropical acompanharam as ações de várias ONGs para chamar atenção de desmatamentos ilegais de florestas tropicais e de promover uma política de desenvolvimento sustentável, novo paradigma do processo pós-Rio 92.

A globalização dos mercados e a demanda por madeira produzida de forma ecologicamente aceitável, sobretudo no exterior, levaram primeiramente as indústrias de painéis reconstituídos do Brasil a estabelecerem uma imagem de empresas ambiental e socialmente responsáveis, a fim de não perderem a competitividade nesses mercados emergentes. Adicionalmente, o desvio da procura de madeira tropical da Ásia para a América do Sul aumentou a necessidade de se adotar uma política conservacionista de exploração da floresta Amazônica.

Uma estratégia para refletir essa nova preocupação, e de ganhar a confiança do consumidor e da opinião pública, é buscar um

\footnotetext{
${ }^{1}$ Engenheiro Florestal, Dr., Administração Florestal do Estado de Baden-Württemberg, Forstdirektion Tübingen, Im Schloss, 72074 Tübingen (Alemanha). Docente na Universidade de Ciências Aplicadas de Rottenburg. Email: Peter.Spathelf@forst.bwl.de,

2 Engenheira Agrônoma, Dra., Pesquisadora da Embrapa Florestas (Email: povoa@cnpf.embrapa.br)

${ }^{3}$ Engenheiro Florestal, Dr., Pesquisador da Embrapa Florestas, Estrada da Ribeira, km 111. Caixa Postal 319 - CEP 83411-000 Colombo (PR), Brasil (Email: botosso@cnpf.embrapa.br)
}

Recebido para publicação: 12/12/2003

Aceito para publicação: 06/12/2004 
diferencial de mercado: a certificação do manejo sustentável de florestas e da origem do produto. Com as informações sobre a sustentabilidade da produção e a garantia de origem dos produtos à disposição dos consumidores, ganha-se espaço nos mercados, difundindo-se entre os produtores a importância e as vantagens de se adotar práticas de bom manejo.

\section{SITUAÇÃO MUNDIAL \\ CERTIFICAÇÃO FLORESTAL}

DA

Entre os sistemas internacionais de certificação florestal destacam-se o FSC (Forest Stewardship Council), fundado em 1993, hoje sediado no México e o PEFC (PanEuropean Forest Certification, desde 2003 Programme for the Endorsement of Forest Certification Schemes), fundada em 1998 como resposta européia à dominância do FSC.

Paralelamente foram introduzidos e, gradativamente, implementados sistemas nacionais de certificação, como o "Finnish Forest Certification System" (Finlândia), "Canadian Standard Association" (Canadá), "Sustainable Forest Initiative" (EUA) e o sistema nacional da Malásia. Na Malásia o conselho nacional estabeleceu critérios e indicadores segundo a ITTO. Na Indonésia há um sistema nacional para concessões, no Brasil o selo CERFLOR, e na Ásia e na bacia do Congo a certificação aprovada pela fundação Kerhout (Eba 'a Atyi \& Simula, 2002).

A área total de floresta certificada no mundo, por todos os sistemas, abrange mais de 120 milhões de hectares, sendo apenas $8 \%$ em países em desenvolvimento. A quantidade de madeira oriunda de florestas certificadas aumentou significativamente nos últimos anos, concentrando-se o marketing dessa madeira em poucos segmentos do mercado internacional. As florestas certificadas pelo FSC no mundo compreendem uma área de mais de 40 milhões de hectares. O maior interesse por produtos com origem certificada, por enquanto, concentra-se em nações européias como os Países Baixos, Alemanha e Reino Unido (FSC, 2003).

\section{CERTIFICAÇÃO FLORESTAL NO BRASIL}

\section{Setor florestal brasileiro}

Com uma área florestal de 544 milhões de ha (FAO, 2001), o Brasil é um dos países mais importantes no mundo em termos de floresta tropical. Dentro da área florestal total, em torno de 5 milhões ha são plantações (entre elas 4,8 milhões são de pinus e eucalipto, situação 2000; SBS, 2003).

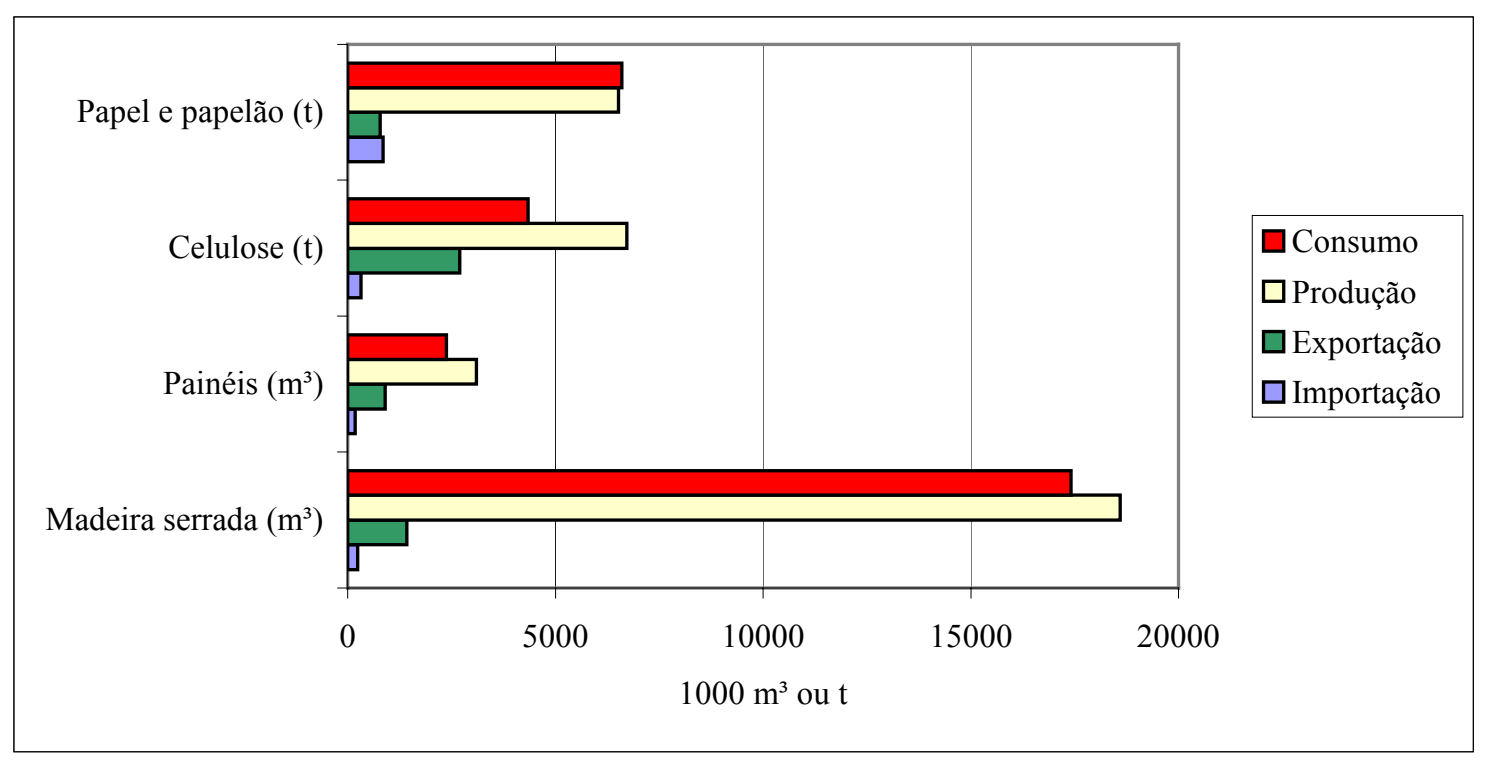

Figura 1: Produção, comércio e consumo de produtos madeireiros no Brasil (FAO, 2001)

Figure 1: Production, trade and consumption of wood products in Brazil (FAO, 2001) 
Além disso, o Brasil é o maior consumidor de madeira tropical no mundo. A madeira é oriunda sobretudo dos "pólos madeireiros" nos estados do Pará, Mato Grosso e Rondônia. Os maiores consumidores internos são as regiões Sul e Sudeste. Só o estado de São Paulo consome mais do que o maior consumidor de madeira tropical na Europa, a França (Seling et al., 2002).

Uma quantidade considerável da madeira produzida para o mercado interno no Brasil consiste em lenha ou madeira para carvão vegetal; essa madeira não será afetada pela certificação no futuro próximo. Sem considerar a lenha, em torno de $95 \%$ da madeira roliça e serrada produzida no Brasil é consumida no mercado interno; $86 \%$ da madeira produzida na Amazônia destina-se ao consumo interno, e $65 \%$ das exportações são compradas pelos EUA e UE, o que corresponde a $2 \%$ da produção de madeira no Brasil. Painéis $(\sim 30 \%)$ e polpa / celulose (40 \%) têm a maior taxa de exportação; a madeira serrada contribui com 7,5\%, e a madeira roliça com menos de $1 \%$ (Fig. 1). A contribuição das exportações brasileiras para o total das exportações de produtos madeireiros no mundo fica em torno de $3 \%$. Constata-se então uma baixa participação do mercado madeireiro brasileiro no mercado internacional (Fig. 2) (FAO, 2001).

O setor da celulose no Brasil pode ser considerado emergente e promissor. Isto se deve às vantagens comparativas na condução de plantações de rápido crescimento e na produção como clima e solo favorável, tecnologia e capacidade industrial de produção.

1000 US \$

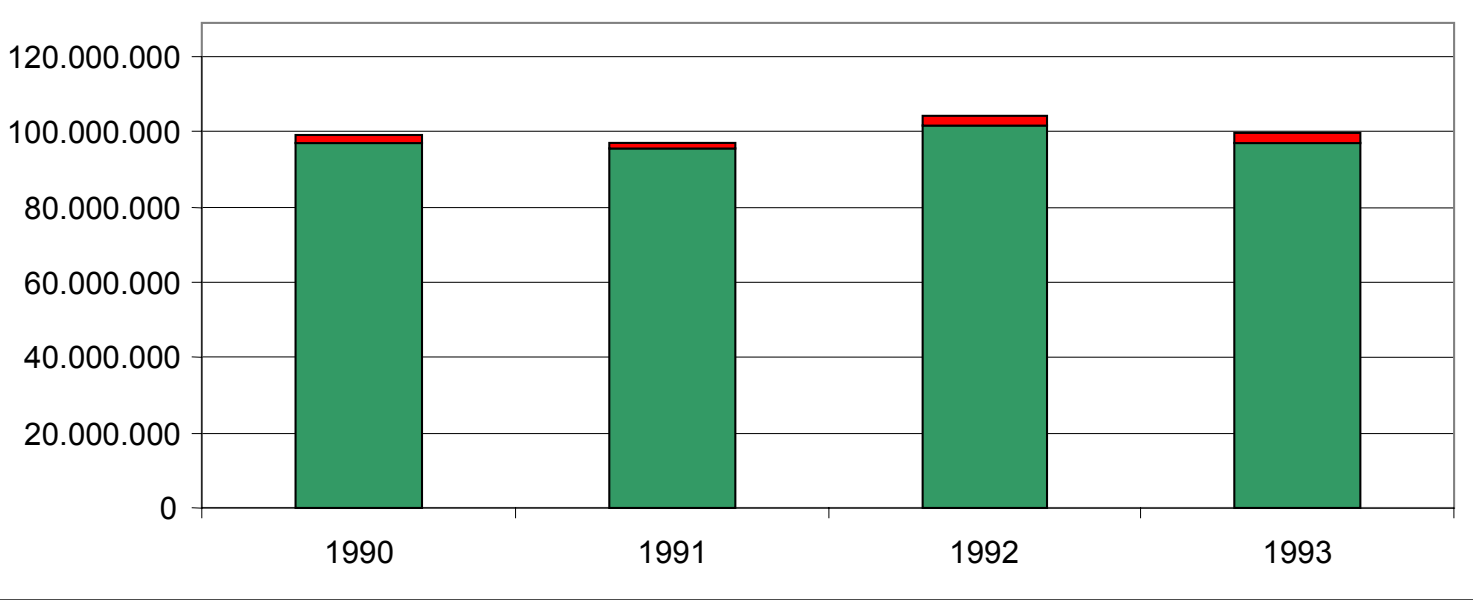

Figura 2: Participação das exportações florestais brasileiras (vermelho) nas exportações mundiais (verde) (IBGE, 1999)

Figure 2: Share of Brazilian forestry related exports (red) at the world exports (green) (IBGE, 1999)

\section{Certificação florestal sob FSC}

A fundação do "FSC Working Group" no Brasil aconteceu em 1997. A tarefa principal foi estabelecer critérios e indicadores para um processo de certificação com base nos princípios do FSC internacional.

A área florestal certificada pelo FSC no Brasil abrange 2,3 milhões de hectares, concentrados em mais de 40 empresas das quais ao redor de 1,3 milhões de hectares são florestas naturais (situação em setembro 2004). A certificação da cadeia de custódia já incorpora mais de 190 empresas com produtos como madeira sólida para móveis e instrumentos musicais.

As principais certificadoras credenciadas pelo FSC no Brasil são: 
- "Rainforest Alliance / Smart Wood Program", EUA; representado no Brasil por Imaflora, SP

- $\quad$ "Scientific Certification System", EUA

- "Société Generale de Surveillance / Qualiflor Program", África do Sul

Elas realizam todos os tipos de certificação, sejam em florestas naturais ou florestas plantadas.

\section{Sistema nacional CERFLOR}

O sistema nacional de certificação CERFLOR foi desenvolvido a partir do início do ano 1993 compatíveis com os critérios da proposta de Tarapoto. Na proposta de Tarapoto dos países vizinhos da Amazônia foram formulados 12 critérios e 37 indicadores de sustentabilidade das florestas na Amazônia, de abrangência global, nacional e referentes a unidade de manejo. A última reunião das partes ocorreu em 2001. Atualmente a tarefa é estender o acordo ao nível nacional, com a proposta de prioridades e sua integração / implementação na política nacional ambiental (Programa Nacional de Florestas).

A iniciativa para um processo nacional de certificação baseia-se em uma parceria da Sociedade Brasileira de Silvicultura (SBS), a Associação Brasileira de Normas Técnicas (ABNT) / Instituto Nacional de Metrologia (INMETRO), Embrapa, Universidades, Empresas e outros interessados. Normas para florestas plantadas já existem tanto como modelos para florestas naturais e a verificação da cadeia de custódia. A Inpacel, uma empresa do grupo International Paper, no Estado do Paraná, foi auditada e recomendada para a certificação florestal pelo sistema CERFLOR. Paralelamente, trabalha-se para o reconhecimento mútuo de sistemas de certificação (ITTO, 2003; SBS, 2003; May \& Vega, 2000).

\section{Grupos de compradores}

Merece destaque os chamados grupos de compradores como peculiaridade no cenário brasileiro de certificação florestal. Os grupos de compradores são integrados por cerca de 60 empresas (entre elas, sobretudo, indústrias, varejistas), estados (Acre, Amapá), uma prefeitura no Estado de São Paulo e dois sindicatos moveleiros. Os grupos de compradores se comprometem a promover madeira certificada já tendo aumentado significativamente a demanda por tal madeira, sobretudo de eucalipto para exportação (FSC, 2003).

A meta para o ano 2005 é comprar 100 $\%$ de madeira certificada, quando oriundas de plantações, e $50 \%$ quando oriundas de florestas naturais. Grupos semelhantes também estão se formando em outros países (May \& Vega, 2000).

\section{CERTIFICAÇÃO FLORESTAL - COMO FUNCIONA?}

É necessário distinguir entre certificação ambiental de produtos (família ISO 14000 , a qual abrange o desenho, a produção de produtos e monitoramento de sistemas producionais sob ponto de vista ambiental), da certificação do manejo florestal (FSC e outros sistemas) e certificação da cadeia de custódia. Esta última, garante a identidade do produto da floresta em toda etapa do processamento. Certificação é um processo voluntário e, no caso da certificação florestal, visa a implementação do "bom manejo florestal". Consiste num pedido de auditoria feita por certificadoras credenciadas, que agem independentemente, mas segundo os princípios e critérios estabelecidos pelo modelo de certificação escolhido.

A madeira extraída da floresta obtém o selo se a floresta for manejada segundo os princípios e critérios do FSC. O processo de certificação, em geral, requer um monitoramento periódico e uma renovação a cada cinco anos. Os custos consistem nos gastos para a auditoria e para a adequação aos padrões do sistema da organização de certificação. Em países em desenvolvimento, os custos para certificação variam entre 0,2 e 1,7 US\$ por ha (Crossley \& Points, 1997).

\section{CONTRIBUIÇÃO DA CERTIFICAÇÃO PARA A CONSERVAÇÃO DAS FLORESTAS NATURAIS}

Uma questão-chave que se levanta é se a certificação representa um instrumento efetivo para diminuir os efeitos negativos da exploração ilegal de madeira em florestas naturais. As forças motrizes mais importantes do desmatamento no Brasil até hoje são (Bruenig, 1996; Burger, 2000; Seling, 2000): 
- opções alternativas economicamente atrativas do uso da terra como a exploração tradicional / predatória de florestas (expansão da fronteira agrícola). É pouco provável que, no futuro, o manejo florestal sustentável se torne economicamente mais atrativo, em comparação à exploração predatória. Pearce et al. (2003) enumeram como fatores para promover um manejo sustentável:

a) preços mais altos da madeira no futuro;

b) taxas de juros mais baixos;

c) títulos de terra mais seguros; e

d) uma situação política mais estável para apoiar empreendimentos / investimentos a longo prazo.

- falta de monitoramento e fiscalização das leis ambientais / florestais vigentes.

- política de planejamento e projetos de infra-estrutura com impactos severos para o uso da terra, também devido à migração de pequenos produtores (Anonymus, 2000).

Ainda existem vários obstáculos para o estabelecimento de um manejo florestal sustentável. Salientam-se as altas exigências da legislação para o manejo de florestas (tropicais) naturais (Bressan et al., 1999; Schneider \& Finger, 2000). Paralelamente, os custos da exploração predatória, na maioria das vezes, são menores do que os com planejamento e técnicas de colheita mais sofisticadas (Pearce et al., 2003).

Além disso, a escassez de conhecimentos científicos quanto à dinâmica de formação das florestais naturais, sobretudo após intervenção, e também a escassez de conhecimentos para mudar de uma exploração predatória para um manejo sustentável, devem ser mencionadas (Schneider \& Finger, 2000). Um manejo florestal sustentável, que compreende a preservação da estrutura dos povoamentos e da diversidade biológica de espécies, além da utilização de uma quantidade contínua de madeira e de produtos nãomadeireiros, ainda não foi comprovado na prática, após os segundo e terceiro ciclos de corte. Sistemas com a intenção do manejo florestal sustentável como o sistema CELOS do Suriname ou o Sistema de manejo sustentado para as florestas brasileiras de terra firme na Amazônia, até hoje, não estão implementados em tempo suficiente para se julgar os impactos sobre a dinâmica e crescimento da floresta, bem como a sustentabilidade econômica (Silva, 2000).

Um primeiro progresso na direção de uma exploração de madeira ou até um manejo florestal mais sustentável é a aplicação das técnicas da colheita florestal de impacto reduzido (RIL $=$ Reduced Impact Logging). $\mathrm{O}$ efeito positivo sobre a utilização da madeira e os baixos danos no povoamento remanescente é evidente e reconhecido, sobretudo em comparação à exploração convencional (ou predatória) - a redução dos prejuízos no povoamento remanescente pode ser de até 50 $\%$. A maioria dos estudos mostra que, a longo prazo, RIL também é mais barato por $\mathrm{m}^{3}$ de madeira colhida do que a colheita florestal convencional (Grammel, 1995; Blate et al., 2001).

A certificação florestal encaixa-se num espectro de medidas para promover o bom manejo de florestas naturais. Do lado da demanda o problema é conscientizar os consumidores / compradores para se obter madeira oriunda de manejo com o selo de certificação, o que pode implicar em um custo diferencial. Devido ao fato da madeira tropical produzida no Brasil ser consumida principalmente no próprio país, precisa-se, sobretudo, considerar o comportamento do consumidor nacional.

A conscientização da população brasileira ainda é pequena, quando comparada à de alguns países com mercados ecologicamente mais sensíveis. Embora perceba-se uma vontade crescente do consumidor brasileiro, pelo menos nos grandes centros urbanos, de considerar aspectos ambientais na decisão da compra, as dúvidas permanecem quanto a aceitação de acréscimo de custo por produtos certificados. Por isso, ainda não há certeza se preços adicionais podem ser realizados a longo prazo. Pesquisas sobre consumidores "premium" internacionais levam ao mesmo resultado (Pearce et al., 2003; Leslie et al., 2002).

No lado da produção, os custos mais altos para estabelecer sistemas de manejo sustentável com certificação, especialmente nos sistemas florestais da Amazônia, requerem 
que a decisão para a certificação seja economicamente justificada pela empresa: por novos mercados conquistados, antigos mercados assegurados, ou o adicional de preço alcançado pelo produto certificado. Para empresas orientadas à exportação é mais provável que esse cálculo se realize. No entanto, para o mercado interno a situação é mais complexa.

\section{NOVAS TENDÊNCIAS}

Como tendência nítida no cenário da certificação florestal internacional, observa-se o aumento das atividades no reconhecimento mútuo entre os principais sistemas. Uma aproximação entre o FSC e o sistema de certificação na Finlândia está em andamento. $O$ PEFC se esforça para o reconhecimento mútuo dos sistemas nacionais. Além disso, uma aproximação entre o PEFC, e o FSC, se torna provável. No Brasil, o CERFLOR busca reconhecimento pelo PEFC. Finalmente, notase uma tendência ao reconhecimento mútuo dos sistemas nos EUA (FAO, 2001).

Paralelo à certificação da madeira, a certificação de produtos não madeireiros tem aumentado a sua importância. Além do certificado da origem de produtos e de sua qualidade, a maneira como estes são produzidos (produtos de comércio justo) terá um papel de destaque no futuro.

\section{CONSIDERAÇÕES FINAIS}

A certificação da produção florestal por si é um instrumento promissor e pode representar um papel catalisador no processo de estabelecimento de um manejo sustentável dos recursos florestais e assim reduzir as altas taxas de desmatamento. As principais vantagens da certificação são :

- alto valor do processo de discussões decorrente das auditorias;

- aumento de transparência e controle da produção florestal, incorporado como precondição para um manejo sustentável;

- fortalecimento de parcerias entre empresas, fornecedores e clientes, pela certificação da cadeia de custódia, melhorando assim o sistema de gerenciamento, como exemplo de controle operacional e de segurança no trabalho.
A certificação ainda não parece a principal força motriz para um manejo sustentável das florestas naturais no Brasil em grande escala, pois até o momento, os produtos certificados atingem apenas uma pequena parte da população. Observa-se no Brasil, como em outras partes do mundo, a dificuldade de se inserir pequenos proprietários no processo de certificação (Mattos et al., 2003).

Nesse contexto, no Brasil, as seguintes medidas são indispensáveis para a conservação das florestas naturais. Parcialmente já são previstas no Programa Nacional Florestal:

- fortalecimento das empresas de extensão rural, pelo treinamento dos técnicos, quanto aos princípios e critérios da certificação florestal, suas vantagens, desvantagens e aplicabilidade;

- fortalecimento de instituições públicas de fiscalização; combate ao uso ilegal de madeira;

- redução da pressão nas florestas naturais pelo estímulo ao reflorestamento de áreas degradadas e do estabelecimento de sistemas de uso sustentável de remanescentes de florestas naturais;

- revisão das normas da legislação florestal relativas ao manejo de florestas naturais, principalmente relacionadas ao ciclo de corte.

\section{REFERÊNCIAS}

ANONYMUS. Projeto pode aumentar desmatamento. Zero Hora, Porto Alegre, 11. de maio 2000.

BRESSAN, D. A., DURLO, M. A. Letra morta. Zero Hora, Porto Alegre, 22. de abril 1999.

BLATE, G. M., PUTZ, F. E. \& ZWEEDE, J. C. Changing harvesting practice in the Amazon. ITTO Tropical Forest Update 11/ 2. 2001. p. 8-9.

BRUENIG, E. Conservation and Management of Tropical Rainforests. An integrated approach to sustainability. CABI, Wallingford. 1996. $339 \mathrm{p}$.

BURGER, D. Der brasilianische Amazonaswald in der internationalen Forst- 
und Entwicklungspolitik. AFZ/Der Wald, Vol. 55, No. 17. 2000. p. 902-905.

CROSSLEY, R. \& POINTS, J. Investing in Tomorrow's Forests: Profitability and Sustainability in the Forest Products Industry. WWF.Godalmung. 1997.

EBA 'A ATYI, R. E. \& SIMULA, M. Forest certification: pending challenges for tropical timber. ITTO Tropical Forest Update 12/3. 2002. p. 5-8.

FAO. State of the world's forests. Rome. 2001. $181 \mathrm{p}$.

FSC. www.fsc.org.br. 2003.

GRAMMEL, R. Entwicklung und Erprobung eines pfleglichen Holzernteverfahrens als Mittel einer nachhaltigen Tropenwaldbewirtschaftung. Project report, Monte Dourado/Jarí, Pará, Brazil. Freiburg, Institute of Forest Utilization and Forestry Work Science. 1995. 26 p.

IBGE (1999): Anuário Estatístico do Brasil 1998.

ITTO. www.itto.org.jp. 2003.

LESLIE, A., SARRE, A, SOBRAL FILHO, M. \& BUANG, A. Forest certification and biodiversity. ITTO Tropical Forest Update 12/3. 2002. p. 13-15.

MAY, P. H. \& VEGA, N. F. C. Barriers to certification of forest management in the Brazilian Amazon: the importance of costs. Rio de Janeiro: Instituto Pró-Natura, International Institute for Environment and Development (IIED), Deutsche Gesellschaft für Technische Zusammenarbeit (GTZ). 2000. www.pronatura.org.br/en/projects.

MATTOS, P. P; SCHAITZA, E. G.; AHRENS, S. Certificação florestal em pequenas propriedades. Colombo: Embrapa Florestas, 2003. 4p. (Comunicado Técnico, 92).

NAMBIAR, E. K. S. Pursuit of Sustainable Plantation Forestry. Southern African Forestry Journal, No. 184. 1999. p. 45-62.

PEARCE, D., PUTZ, F. E. \& VANCLAY, J. K. Sustainable foretry $n$ the tropics: panacea or folly? Forest Ecology and Management 172. 2003. p. 229-247.

SBS. www.sbs.org.br. 2003.

SCHNEIDER, P. R., FINGER, C.A.G. Manejo sustentado de florestas inequiâneas heterogêneas. Universidade Federal de Santa Maria, Centro de Pesquisas Florestais. 2000. $174 \mathrm{p}$.

SELING, I. 500 Jahre brasilianische Waldgeschichte - Kolonisierung und Tropenwaldzerstörung. AFZ/Der Wald, Vol. 55, No. 17. 2000. p. 884-886.

SELING, I., KAISER, B. \& SPATHELF, P. Chancen der Vermarktung von zertifiziertem Holz auf dem inländischen Markt in Tropenwaldländern am Beispiel Brasilien. Forstarchiv 73/1. 2002. p. 23-29.

SILVA, J. N. M. Manejo de florestas tropicais: o caso da Amazônia brasileira. $1^{\circ}$ Simpósio Latino-Americano sobre Manejo Florestal, 9./10. novembro 2000, Santa Maria, Rio Grande do Sul, Brasil. 2000. p. 15-28. 